Revue d'études intertextuelles et intermédiales

\title{
Album de famille ou roman familial? Transpositions génériques et vacillations sémantiques dans Family and Friends de Anita Brookner
}

Laurence Petit

\section{CpenEdition}

Journals

Édition électronique

URL : http://journals.openedition.org/polysemes/686

DOI : $10.4000 /$ polysemes.686

ISSN : 2496-4212

Éditeur

SAIT

Édition imprimée

Date de publication : 1 janvier 2012

Pagination : 267-283

ISSN : 0999-4203

\section{Référence électronique}

Laurence Petit, «Album de famille ou roman familial ? Transpositions génériques et vacillations sémantiques dans Family and Friends de Anita Brookner », Polysèmes [En ligne], 12 | 2012, mis en ligne le 01 mars 2015, consulté le 21 avril 2019. URL : http://journals.openedition.org/polysemes/686 : DOI : 10.4000/polysemes.686

Ce document a été généré automatiquement le 21 avril 2019.

Polysèmes 


\title{
Album de famille ou roman familial ? Transpositions génériques et vacillations sémantiques dans Family and Friends de Anita Brookner
}

\author{
Laurence Petit
}

1 Comme l'explique Marianne Hirsch dans Family Frames: Photography, Narrative and Postmemory ${ }^{1}$, les photos de famille, qui soulignent la cohésion familiale à travers les poses conventionnelles qu'elles adoptent, perpétuent des schémas familiaux dominants souvent très éloignés de la réalité telle qu'elle est vécue. Ces photographies véhiculent par conséquent «un regard familial externe» (Hirsch parle de «familial gaze $»^{2}$ ) institutionnalisé qui reflète les stratégies familiales d'auto-représentation. Les textes métaphotographiques, qui transposent des photos de famille dans des contextes narratifs soit en les reproduisant matériellement, soit en les décrivant ekphrastiquement, sapent et dénoncent ces idéologies familiales, révélant ce que Benjamin dans «L'œuvre d'art à l'âge de la reproduction mécanique " appelle "l'inconscient optique $»^{3}$ des photos de famille, et faisant resurgir les leurres et les tractations souvent compliquées de la vie familiale. Ces stratégies narratives subversives qui déstabilisent les modes traditionnels d'auto-représentation familiale appartiennent à ce que Hirsch appelle « le regard familial interne» («the familial look»), qui interroge la photographie et en perce la surface plane.

2 Le cinquième roman de Anita Brookner, Family and Friends ${ }^{4}$, constitue précisément un exemple de texte métaphotographique en étant ce que Brent McLaine appelle un « roman album de famille» («a family album novel $»^{5}$ ), sous-catégorie distincte des romans combinant photographies et récit, ou ce que Hirsch, qui a forgé l'expression « photographies en prose» ( " prose pictures $\left.»^{6}\right)$, appellerait par extension « un album de famille en prose » («a prose family album »). Pour McLaine, les romans album de famille, comme la plupart des photofictions, "explore[nt] la tension entre les dimensions simultanément factuelle et interprétative des photographies $»^{7}$. Family and Friends, à la 
frontière des deux codes sémiotiques distincts du texte et de l'image, présente cette tension en mettant en scène un narrateur anonyme scrutant un album de photos de mariage fictives et révélant progressivement - sans toutefois jamais le désigner explicitement - un secret de famille ignoré de tous, ou tout du moins farouchement gardé.

3 Dans les pages qui suivent, je me propose de combiner le concept freudien de « roman familial » (ce fantasme dans lequel un enfant remplace ses parents par d'autres mieux nés) et la distinction qu'opère Hirsch entre « regard familial externe » et « regard familial interne» pour montrer comment cette saga ou roman d'une famille peut se lire ironiquement comme un «roman familial» inversé au sens freudien, dans lequel le narrateur découvrirait, au terme de sa quête, sa propre bâtardise, réelle cette fois, et non pas seulement fantasmée. La transposition ou oscillation générique sur laquelle repose ce roman s'accompagne donc dans le même temps d'une vacillation ou oscillation sémantique renforcée par l'ambiguïté du narrateur.

Par le biais d'une stratégie narrative originale combinant les deux codes sémiotiques du texte et de l'image, Family and Friends se présente sous la forme d'un album de photos de famille fictif dans lequel sont regroupées des photos de mariage couvrant trois générations. Le roman débute et s'achève par la présence d'un narrateur qui ouvre, parcourt, puis referme l'album de photos, procédé qui lui permet au passage de raconter la saga des Dorn, une famille d'exilés juifs ayant fui l'Allemagne nazie pour aller trouver refuge à Londres, puis en Italie et en Californie pour deux de ses membres.

Puisque chaque photo fictive est présentée par le biais d'injonctions narratoriales invitant le lecteur à contempler des images photographiques plutôt qu'à lire des mots bien que ces photos ne se matérialisent jamais et n'aient qu'une existence purement textuelle -, le lecteur est encouragé à jouer le jeu de cette fiction, c'est-à-dire à suspendre tout doute (" suspend his disbelief ») et à percevoir cet objet littéraire hybride comme un album de photos et non un roman : "Here's Sofka, in a wedding-photograph", lit-on dès la première phrase du roman (7) ; « Here they all are, family and friends, in the wedding photograph ", lit-on au début du tout dernier paragraphe (187). Le cheminement visuel à travers l'album est effectué par ce narrateur, ou plutôt par cette « voix » narrative qui, malgré ses nombreuses et péremptoires intrusions à la première personne, demeure néanmoins anonyme et par conséquent énigmatique tout au long du roman, tout en prétendant pourtant avoir, ou avoir eu, des liens privilégiés avec la famille présentée dans l'album. Cet exemple de ce que Marianne Hirsch appelle « une post-mémoire secondaire, indirecte et différée $\aleph^{8}$, dont la médiation est assurée par un narrateur ambivalent qui à la fois témoigne d'un vif intérêt pour l'histoire de cette famille et dans le même temps garde ses distances, est, selon elle, caractéristique de la condition postmoderne dans laquelle les photos de famille deviennent l'élément qui fournit une illusion de continuité dans le temps et dans l'espace au sein de vies façonnées par l'exil, l'émigration et le déracinement. Les quatre photos de mariage qui ponctuent Family and Friends et qui figent de temps à autre le flux narratif avant de le relâcher à nouveau constituent, en quelque sorte, les piliers temporels et structurels sur lesquels repose le récit.

6 Le roman s'ouvre sur une photo de mariage mettant en relief la figure matriarcale de Sofka Dorn, entourée de ses quatre enfants, ses deux filles, Mimi et Betty, et ses deux fils, Alfred et Frederick. Après un premier chapitre consacré largement à la description de cette photo inaugurale, au personnage central de la mère, Sofka, et aux festivités de cette 
journée-là, la narration reprend un à un les personnages principaux de la photo et suit leurs destinées respectives de l'enfance à l'âge mûr, par le procédé photographique ou cinématographique du «zoom» ou mouvement téléscopique permettant de passer du plan général ou panorama de la première photo au gros plan sur un personnage particulier. Par souci de réalisme, puisque le lecteur est censé feuilleter un album de photos s'étalant sur une trentaine ou une quarantaine d'années, chaque chapitre envisage chaque personnage à un moment de sa vie différant de celui choisi pour ses autres frères et sœurs, et ce procédé contribue largement à la grande fluidité linéaire et temporelle du roman, en dépit de l'apparent morcellement qu'implique la structure d'ensemble.

Une étude plus approfondie de ces quatre photographies révèlerait cependant qu'elles peuvent toutes se lire comme des variations anamorphiques de la matrice originelle éternellement répétée et reproduite textuellement. Cette façon d'envisager l'ensemble du roman comme un vaste processus "anamorphotique " ou anamorphique nous parait convaincante si l'on cesse de considérer que le narrateur nous présente des photos pour y voir au contraire une photo, toujours la même, qui se déploierait, tel un éventail, à partir de la première page, et prendrait, au fil de la lecture-déplacement, des apparences nouvelles. Car force est de reconnaitre que ces photos de mariage appartiennent toujours au même genre, le portrait de famille conventionnel, présentent toujours les membres de la famille dans la même position, regroupés autour des jeunes mariés, et surtout présentent toujours les mêmes personnages, Sofka, ses enfants, et le reste de la famille. Les structures fondamentales, immuables, de la première photo sont donc bien respectées, et seuls changent les visages des protagonistes par une sorte de rotation interne faisant que chacun occupe tour à tour la position centrale de marié, de matriarche, de proche, etc. Mais au fond, c'est toujours la même photo qui nous est présentée, et les photos qui se succèdent ne sont jamais que des reproductions, ou plutôt ici des «réécritures", de la première photo séminale, que l'on peut qualifier de photo «matrice ", et ce d'autant plus légitimement qu'elle figure, avec Sofka, la matriarche par excellence.

8 La mise en évidence de cette nouvelle structure d'ensemble de Family and Friends par le truchement de l'anamorphose n'en rend que plus suspecte l'insistance que manifeste le narrateur à vouloir nous faire visualiser de façon répétée ce qui n'est au fond qu'une seule et même photo. N'est-ce pas là la preuve que celle-ci lui fait «signe» (et en cela cesse d'être un simple "référent »), le hante, le fascine, et fonctionne en somme comme ces tableaux « pièges à regard » décrits par Lacan dans Les Quatre Concepts fondamentaux de la psychanalyses, par où s'engouffrent le désir et le fantasme ? On est là au carrefour de ce que Barthes appelle, dans L'Obvie et l'obtus, la fonction « haptique » du regard, c'est-à-dire de cette faculté qu'a le regard de «saisir » et «d'être saisi» en retour ${ }^{10}$, ainsi que du fameux « Ça me regarde » de Lacan. Sofka et les photos qui la convoquent au sein de la narration sont en effet au cœur de ce qui s'apparente à une sorte de quête du narrateur prenant la forme d'une lecture interprétative des photos à la première personne, ou " exégèse lyrique ", pour reprendre l'expression utilisée par Jefferson Hunter ${ }^{11}$ au sujet de La Chambre claire de Barthes ${ }^{12}$. Ce qui commence par une rencontre visuelle avec une photo contenue dans un album se transforme bientôt en une vaste entreprise de réminiscence, d'anamnèse, et la quête ayant pour origine le regard se fait peu à peu discursive, l'ordre du spéculaire - c'est-à-dire ici l'ordre du voir, selon l'étymologie latine speculare signifiant « observer » - laissant progressivement place à l'ordre du spéculatif. La photo devient alors un outil doublement heuristique, tant pour le narrateur dans sa 
quête mystérieuse d'un sens à trouver derrière la surface lisse de ces images, que pour le lecteur, embarqué dans une quête parallèle pour en savoir davantage sur ce mystérieux narrateur.

9 La vue de la première photo de l'album provoque chez le narrateur un véritable choc qui a pour conséquence de fausser la fiabilité de ses perceptions, comme le montre l'erreur qu'il commet en pensant dans un premier temps que Sofka est au centre de la première photo de mariage, et non les jeunes mariés. La photo peu à peu "révélée » par la description est alors elle-même "révélation ", et ce doublement, car si elle révèle au narrateur un sens, ou une esquisse de sens, qui le pousse à se lancer dans son entreprise de décryptage en profondeur de l'image, elle est aussi le facteur qui révèle au lecteur le narrateur lui-même. La présence de ce dernier devient en effet de plus en plus manifeste à mesure qu'il intervient ostensiblement dans la narration à l'occasion de chaque description de photo. Or la description est précisément le lieu, comme le souligne Philippe Hamon dans Du Descriptif, d'une " ostentation du sujet à travers un sujet ${ }^{13}$ ou, devrait-on plutôt dire, d'une " ostentation du sujet à travers un objet », l'objet étant en l'occurrence les photos de mariage. Cette volonté du narrateur de diriger, voire de manipuler, le paysage mental du lecteur a en effet pour conséquence de se retourner contre lui et de susciter chez le lecteur intrigué un désir d'en savoir davantage sur ce mystérieux narrateur qui, paradoxalement, se dérobe alors même qu'il dévoile toujours davantage de faits. Il y a par conséquent un décalage constant entre ce que l'on nous dit de voir et ce que nous souhaitons voir.

Plus qu'une entreprise élégiaque fortement teintée de nostalgie à la gloire d'un personnage phare (ici la défunte matriarche), processus relativement courant dans la " photofiction » si l'on en croit Brent MacLaine ${ }^{14}$, cette quête du narrateur nous semble ici s'apparenter davantage à l'écriture de son propre « roman familial », tel que Freud décrit ce mythe dans « Le roman familial des névrosés $»^{15}$ et que Marthe Robert le reprend dans son ouvrage Roman des origines et origines du roman ${ }^{16}$. Le « roman familial » est ce fantasme particulier dans lequel le sujet s'imagine être né de parents différents des siens et généralement de rang social plus élevé. Comme l'écrit Marthe Robert :

C'est ainsi que [l'enfant] en vient à se raconter des histoires, ou plutôt une histoire qui n'est rien d'autre qu'un arrangement tendancieux de la sienne, une fable biographique conçue tout exprès pour expliquer l'inexplicable honte d'être mal né, mal loti, mal aimé [...]. Devenus méconnaissables à ses yeux depuis qu'il leur découvre un visage humain, ses parents lui paraissent tellement changés qu'il ne peut plus les reconnaître pour siens. Il en conclut que ce ne sont pas ses vrais parents, mais littéralement des étrangers, des gens quelconques avec lesquels il n'a rien de commun si ce n'est qu'ils l'ont recueilli et élevé. Ayant ainsi interprété le sentiment d'étrangeté que lui inspirent maintenant ses anciennes idoles démasquées, il peut désormais se regarder comme un enfant trouvé, ou adopté, auquel sa vraie famille, royale, bien entendu, ou noble, ou puissante en quelque façon, se révélera un jour avec éclat pour le mettre enfin à son rang. ${ }^{17}$

11 Bien qu'il ne soit jamais question ouvertement de la part du narrateur d'une quelconque démarche autobiographique, plusieurs indices laissent à penser qu'une telle entreprise pourrait bien être ce qui sous-tend ce parcours visuel de l'album de photos, à commencer par l'aspect résolument généalogique du roman, s'étalant sur trois générations, soit celle des grands-parents, avec Sofka, celles des parents, avec Mimi, Betty, Frederick et Alfred, et celle des enfants, ceux de Frederick, par exemple, ou ceux qui apparaissent sur la dernière photo qui clôt le roman, à savoir Laurie, Charlie, et Vicky. En fait, une indication paratextuelle d'importance révèle que Anita Brookner s'est inspirée de photographies de 
membres de sa propre famille, de la génération de ses parents et grands-parents, ce qui suggérerait que le narrateur, si l'on part du présupposé que celui-ci est contemporain de Brookner, appartiendrait à la génération de ceux que l'on vient de décrire comme les «enfants» dans le roman. Dans l'entretien qu'elle accorda à Olga Kenyon en 1989, Brookner révéla qu'une photo de mariage de sa propre grand-mère était à l'origine de ce roman $^{18}$. Nous avons là par conséquent le cas intéressant d'un roman dont l'origine génétique est fidèlement reproduite dans l'incipit, et dont la genèse même est présentée par Brookner comme la mise en fiction d'une réalité déjà en grande partie fictive :

To a certain extent, the early lives of these uncles and aunts are fictitious, for I knew nothing of their early lives and was obliged to invent them. [...] Whether this was an obscure form of unconscious memory, whether it was intuition, or whether it was the exhilaration of disposing of these characters whom I had always seen as immensely powerful, I have no idea. ${ }^{19}$

Et pourtant, c'est essentiellement la fin très ambiguë de Family and Friends qui nous permet de considérer l'ensemble du roman non seulement comme la saga ou le roman d'une famille, mais plutôt comme un « roman familial» au sens freudien. Cette fin, qui consiste en une ekphrasis de la dernière photo de mariage de l'album, est à ce titre extrêmement troublante, car si l'ensemble du roman, sous prétexte de nous " faire voir ", ne cesse en fait de nous "dire" les choses, la description finale fonctionne essentiellement sur le "non-dit ", tout en nous donnant énormément à " voir ", dans ce qui ressemble fort à un clin d'œil appuyé du narrateur au lecteur. Mais voyons plutôt le passage en question :

Here they all are, family and friends, in the wedding photograph. It is the last one in the album. George and Ursie stand, politely smiling, between Lili and Mrs Beck. Dolly, slightly out of focus, as she was in reality on that day, appears to lean heavily on Hal. Will smiles, plump, good-natured, unquestioning as ever. Mimi, upright, in pale lace, with a rather imposing hat, looks very like her mother. Lautner, although greatly diminished, still turns to her fondly. Here is Alfred, tall, stiff, still a handsome man. Here is Nettie, very close to Alfred, leaving Will almost unattached, unpaired. And in the front row, the three children: Laurie, Charlie, and Nettie's child Vicky (Victoria). See that look on Vicky's face, that imperious stare, so unlike a child, so like Sofka. See Alfred's hand proudly clasping her little shoulder. See the resemblance. Wait for the dancing to begin. (187)

13 Une lecture attentive de ce passage permet de dégager une structure binaire dans laquelle les personnages apparaissent par paire, correspondant le plus souvent à des couples mariés, comme le couple que forment Ursie et George, ou Dolly et Hal, ou encore celui constitué par Mimi et Lautner. Ces couples répondent parfaitement aux exigences de la pose officielle de la photo de mariage, soit à ce que Mariane Hirsch appelle le « regard familial externe» («the familial gaze») institutionnalisé. Une "paire» pose toutefois problème : il s'agit de celle constituée par Alfred et Nettie, qui est suggérée par le parallélisme grammatical « Here is Alfred », « Here is Nettie », le rapprochement de ces deux locutions dans deux phrases consécutives, et la proximité effective des deux personnages dans la photo, Nettie se tenant "très près d'Alfred" ("very close to Alfred »). Par ailleurs, le fait même de les désigner, au cœur de la description, par deux énonciations déictiques commençant par «Here " semble les mettre en relief comme si c'étaient eux les jeunes mariés occupant la position centrale de la photo. Or il n'en est rien, nous le savons : le marié et la mariée se trouvent être George et Ursie "smiling politely ", Nettie est elle-même mariée à Will, et Alfred est un célibataire endurci. Ce que la photo suggère en revanche, c'est une relation adultère entre Nettie et Alfred, l'amant 
notoire des femmes mariées (comme l'a montré précédemment dans le roman sa longue liaison avec sa cousine Dolly). Cette impression est d'ailleurs corroborée par l'insistance du narrateur à souligner la solitude anormale de Will, «almost unattached, unpaired " (alors qu'il est justement lié à Nettie par le mariage), et la préférence de Nettie pour Alfred avec l'utilisation pour le moins ambiguë du verbe «to leave » dans l'expression «leaving Will almost unattached». De plus, Will est décrit par le narrateur, avec une certaine ironie, comme "plump, good-natured, unquestioning as ever", ce qui laisse à penser que cette discrétion de sa part pourrait bien être soit de la naïveté, soit une sorte de consentement tacite.

Mais ce que suggère surtout la photo, c'est qu'une enfant est le fruit de cette union illégitime : il s'agit de Vicky, présentée comme «l'enfant de Nettie» (et non comme la fille de Nettie et de Will), et dont le père semble n'être autre qu'Alfred lui-même, car sinon pourquoi la main de ce dernier serrerait-elle « fièrement » l'épaule de la fillette ? Et pourquoi le narrateur chercherait-il, par une nouvelle série d'anaphores phatiques, à nous faire voir la « ressemblance » qui existe à la fois entre Vicky et Alfred, et entre Vicky et Sofka, la grand-mère qu'elle ne connaîtra jamais? (« See that look on Vicky's face, that imperious stare, so unlike a child, so like Sofka. See Alfred's hand proudly clasping her little shoulder. See the resemblance »).

On peut dès lors avancer l'hypothèse que ce mystérieux narrateur, qui nous retrace avec tant de passion l'histoire de Sofka et de ses descendants, est précisément la petite Vicky qui, devenue adulte, partirait, à travers l'album de photos, en quête de ses racines, à la recherche d'une vérité non pas tant sur Sofka et les siens, mais bien sur elle-même. En ce sens, sa façon de scruter les photographies correspond tout à fait à ce que Marianne Hirsch appelle «le regard familial interne» («the familial look»), de nature investigatrice et subversive. La fiction que nous présente Family and Friends peut ainsi se lire comme la propre fiction du narrateur, celle de ses origines, et l'ironie veut qu'en écrivant ce "roman d'une famille», en l'occurrence de sa famille, la narratrice découvre, au terme de sa quête, qu'il s'agit bel et bien d'un «roman familial » au sens où Freud l'entend, puisqu'elle est réellement une enfant bâtarde. Mais on a là en quelque sorte un « roman familial inversé », puisque la narratrice ne s'invente pas une fable biographique dans laquelle elle serait une enfant bâtarde, comme dans tout "roman familial» classique, mais découvre, au terme d'une quête aux accents autobiographiques, qu'elle l'est réellement.

16 Le rapprochement effectué entre Family and Friends et le "roman familial» tel que le décrivent Freud et Marthe Robert paraît légitime dans la mesure où Anita Brookner ellemême montre une parfaite connaissance de ces théories psychanalytiques, au point d'intituler son treizième roman A Family Romance ${ }^{20}$ et d'y écrire la phrase suivante : «I never felt excluded from their lives, never witnessed any primal scene, was not encouraged to formulate any family romance, although I was to do this later in the books I wrote for children and for which I became quite well-known » (44). « Family romance » est l'expression anglaise utilisée en psychanalyse pour faire référence au "roman familial ». On a aussi bien entendu dans ce titre un jeu sur les mots "family " et " romance ». Le roman raconte la fascination de la narratrice, Jane, pour sa tante par alliance, Dolly, et en dépit de ce que prétend la narratrice dans l'extrait ci-dessus, cette " romance » familiale va peu à peu se transformer en « roman familial », Jane s'inventant en Dolly une seconde mère, à la vie plus palpitante que celle de sa vraie mère, et Dolly finissant par considérer Jane comme sa fille et, ce faisant, par s'inventer la fille qu'elle n'a 
jamais eue. Family and Friends, en revanche, peut être considéré comme un roman familial inversé en ce que le "regard familial externe » conventionnel et institutionnel de ces photos de mariage interchangeables est progressivement sapé par le «regard familial interne » investigateur, voire inquisiteur, ou plutôt, devrions-nous dire, par le « regard narratorial interne » d'un narrateur dont l'identité subversive est finalement révélée, ou plutôt discrètement suggérée, à la toute fin du roman.

L'écart qui existe entre d'un côté, les stratégies familiales officielles d'auto-représentation reflétées par ces photos de mariage éminemment conventionnelles, et de l'autre, le secret ignoré de tous, ou tout du moins bien gardé, que recèle la toute dernière photo, n'illustre pas seulement la façon dont les albums de famille perpétuent et déstabilisent tout à la fois l'idéologie familiale. Plus généralement, cet écart rappelle les remarques de Deborah Bowen sur l'utilisation ironique que fait Brookner de la photographie dans ce roman album de famille, en soulignant la « sécurité apparente et pourtant trompeuse " de ces photographies («the apparent but deceptive security of these photographs ») ${ }^{21}$. En ce sens, par le biais des vacillations sémantiques que produit cette transposition générique, Family and Friends va au-delà des interrogations postmodernes « classiques » concernant la représentation littéraire et ses limites pour amorcer une réflexion sur des notions-clés, pour qui s'intéresse aux interactions entre textes et images, telles que l'immédiateté, l'artifice, la stabilité, les "évidences» visuelles. À l'instar de nombreux écrivains postmodernes, Anita Brookner, qui fut longtemps historienne de l'art, explore le pouvoir déstabilisant des images et leur capacité à remettre en cause les concepts de perception et de représentation.

\section{BIBLIOGRAPHIE}

Barthes, Roland. La Chambre claire. Notes sur la photographie. Paris : Gallimard, 1980.

Barthes, Roland. L'Obvie et l'obtus. Essais critiques III. Paris : Seuil, coll. Tel, 1982.

Benjamin, Walter. "The Work of Art in the Age of Mechanical Reproduction" (1935). Illuminations. New York: Schocken Books, 1968.

Bowen, Deborah. "Preserving Appearances: Photography and the Postmodern Realism of Anita Brookner". Mosaic, A Journal for the Interdisciplinary Study of Literature, University of Manitoba, 28.2 (June 1995): 123-148.

Brookner, Anita. A Family Romance. London: Jonathan Cape, 1993.

Brookner, Anita. Family and Friends. London: Jonathan Cape, 1985.

Freud, Sigmund. «Le roman familial des névrosés ». Névrose, psychose et dépression. Paris : PUF, 1973.

Hamon, Philippe. Du Descriptif. Paris : Hachette, 1993.

Hirsch, Marianne (ed.). Family Frames: Photography, Narrative and Postmemory. Cambridge, Mass.: Harvard UP, 1997. 
Hirsch, Marianne (ed.). The Familial Gaze. Hanover, New England: UP of New England, 1999.

Hunter, Jefferson. Image and Word: the Interaction of 20th Century Photographs and Texts. Cambridge, Mass.: Harvard UP, 1987.

Kenyon, Olga. “Anita Brookner”. Women Writers Talk: Interviews with 10 Women Writers. Oxford: Lennard Publishing, 1989.

Lacan, Jacques. Le Séminaire livre XI. Les Quatre Concepts fondamentaux de la psychanalyse (1964).

Paris : Seuil, 1973.

McLaine, Brent. "Photofiction as Family Album: David Galloway, Paul Theroux and Anita Brookner". Mosaic, A Journal for the Interdisciplinary Study of Literature, University of Manitoba, 24.2 (Spring 1991): 131-149.

Robert, Marthe. Roman des origines et origines du roman. Paris : Grasset, 1972.

\section{NOTES}

1. Marianne Hirsch (ed.) Family Frames: Photography, Narrative and Postmemory, Cambridge, Mass.: Harvard UP, 1997.

2. Marianne Hirsch (ed.), The Familial Gaze, Hanover, New England: UP of New England, 1999.

3. Walter Benjamin, "The Work of Art in the Age of Mechanical Reproduction" (1935), Illuminations, New York: Schocken Books, 1968, 237.

4. Anita Brookner, Family and Friends, London: Jonathan Cape, 1985. Toutes les références entre parenthèses dans le texte se réfèrent à cette édition.

5. Brent McLaine, "Photofiction as Family Album: David Galloway, Paul Theroux and Anita Brookner", Mosaic, A Journal for the Interdisciplinary Study of Literature, University of Manitoba, 24.2 (Spring 1991): 131.

6. Hirsch, The Familial Gaze, op. cit.

7. McLaine, op. cit., 131 : «Among the large number of novels which combine photography with narrative-either by actually including photographs with texts [...], or simply by describing photographs [...]-novels which pretend to be family photograph albums define a recognizable subgenre. Family album novels, like most photofictions, explore the tension between the simultaneously factual and interpretative qualities of photographs ».

8. Hirsch, Family Frames, op. cit. : " a delayed, indirect and secondary postmemory ».

9. Jacques Lacan, Le Séminaire livre XI. Les Quatre Concepts fondamentaux de la psychanalyse (1964), Paris : Seuil, 1973, 102.

10. Roland Barthes, L'Obvie et l'obtus. Essais critiques III, Paris : Seuil, coll. Tel, 1982, 279.

11. Jefferson Hunter, Image and Word: the Interaction of 20th Century Photographs and Texts, Cambridge, Mass.: Harvard UP, 1987, 164.

12. Roland Barthes, La Chambre claire. Notes sur la photographie, Paris : Gallimard, 1980.

13. Philippe Hamon, Du Descriptif, Paris : Hachette, 1993, 17.

14. McLaine, op. cit., 132 : «While the evidential nature of the photograph has from the beginning been aligned with its supposed ability to stabilize the world "realistically", its destabilizing or transforming function, as family album novels show, is more variously aligned with a number of compromising factors: the photographer's selection and manipulation of the image, the subject's posturing, private or privileged knowledge, context, nostalgia, memory, artfulness and elegiac purpose ».

15. Sigmund Freud, «Le roman familial des névrosés », Névrose, psychose et dépression, Paris : PUF, 1973. 
16. Marthe Robert, Roman des origines et origines du roman, Paris : Grasset, 1972.

17. Ibid., 46-47.

18. Olga Kenyon, “Anita Brookner”, Women Writers Talk: Interviews with 10 Women Writers, Oxford: Lennard Publishing, 1989.

19. Ibid., 16.

20. Anita Brookner, A Family Romance, London: Jonathan Cape, 1993

21. Deborah Bowen, "Preserving Appearances: Photography and the Postmodern Realism of Anita Brookner", Mosaic, University of Manitoba, 28.2 (June 1995): 139.

INDEX

oeuvrecitee Family and Friends 